\title{
CHEMOSPHERE
}

\section{Formation of PCDD/Fs and PCBs in the process of production of 1,4-dichlorobenzene}

\author{
Wenbin Liu, Minghui Zheng *, Dongsheng Wang, Ying Xing, \\ Xingru Zhao, Xiaodong Ma, Yong Qian
}

Key Laboratory of Environmental Chemistry and Ecotoxicology, Research Center for Eco-Environmental Sciences, Chinese Academy of Sciences, P.O. Box 2871, Beijing 100085, China

Received 3 February 2004; received in revised form 24 August 2004; accepted 9 September 2004

\begin{abstract}
The occurrence and distribution of polychlorinated dibenzo-p-dioxins (PCDDs), polychlorinated dibenzofurans (PCDFs) and polychlorinated biphenyls (PCBs) in a typical process of production of 1,4-dichlorobenzene (p-DCB) were investigated systematically in this study. $\mathrm{P}_{5} \mathrm{CDFs}$ and $\mathrm{H}_{6} \mathrm{CDFs}$ were the main congeners of PCDD/Fs, while only low levels of other PCDD/Fs were detected in the samples. The concentrations of PCBs were up to $4614 \mathrm{ng} / \mathrm{g}$ in the semi-manufactured products, and $1797 \mathrm{ng} / \mathrm{g}$ in the product p-DCB. It was also found that high chlorinated PCB congeners increased with the further chlorination. The levels of PCDD/Fs decreased obviously in the purification process, while PCBs could not be removed effectively. Polychlorophenols and other important dioxin precursors (1,4-dichloro-2phenoxybenzene and 2-phenyl-2',5,5'-trichlorophenol) were also analyzed. Polychlorophenols which ortho- and parapositions of the hydroxyl were substituted by chlorine were the main congeners. In addition, a possible pathway of the formation of PCDD/Fs and PCBs in the production of $\mathrm{p}-\mathrm{DCB}$ was presented. The results of this article suggest that re-estimation on the risk of using p-DCB products, especially the products for daily use such as mothballs, is expected. (c) 2004 Elsevier Ltd. All rights reserved.
\end{abstract}

Keywords: PCDD/Fs; PCBs; Mothball; p-DCB; Dioxin precursors; Production

\section{Introduction}

Polychlorinated dibenzo-p-dioxins (PCDDs), polychlorinated dibenzofurans (PCDFs) and polychlorinated biphenyls (PCBs) are among the persistent organic pollutants targeted for international source

\footnotetext{
* Corresponding author. Tel.: +86106284 9172; fax: +8610 62923563.

E-mail address: zhengmh@mail.rcees.ac.cn (M. Zheng).
}

reduction by the Stockholm Convention. Exposure to PCDD/Fs and PCBs is of great concern because of their toxicity, which would induce hormone-dependent cancers and reproductive effects in humans and wildlife. PCDD/Fs are ubiquitous environmental contaminants, and several of the PCDD/Fs congeners, particularly those substituted at 2,3,7,8-positions, are persistent and bioaccumulative. Recently, special attention has been focused on a few select PCB congeners which are stereochemically similar to 2,3,7,8-substitude PCDD/ Fs. The World Health Organization has identified 12 of the 209 possible PCBs as being similar in toxicity to 
the PCDD/Fs. The list of these $12 \mathrm{PCBs}$ includes four non-ortho (IUPAC nos. 77, 81, 126, and 169) and eight mono-ortho congeners (IUPAC nos. 105, 114, 118, 123, 156, 157, 167, and 189).

The primary sources of PCDD/Fs have been identified in many countries as combustion processes such as waste incineration and various chemical processes where chlorine is involved. In particular, chemicals such as PCBs, pentachlorophenol, 2,4-dichlorophenoxy acetic acid, 2,4,5-trichlorophenoxyacetic acid, and 2,3,5,6tetrachloro-2,5-cyclohexadiene- 1,4-dione were found to be contaminated with PCDD/Fs (UNEP, 2003).

The chemical 1,4-dichlorobenzene (p-DCB), also called paramoth, is one of the chemicals commonly used to make mothballs. For the more than 20 years, p-DCB has been used principally (35-55\% of all uses) as a space deodorant for toilets and refuse containers, and as a fumigant for control of moths, molds, and mildews (HSDB, 1998). The process of $\mathrm{p}-\mathrm{DCB}$ production currently used in industry is direct chlorination of benzene or chlorobenzene in the presence of a Friedel-Crafts catalyst. The usual catalyst is ferric chloride $\left(\mathrm{FeCl}_{3}\right)$, which can be added to the reaction mixture or generated in situ by exposing a large iron surface to the liquid being chlorinated. In addition to p-DCB, the other major isomer of ortho-dichlorobenzene, and a very small amount of $m e t a$-isomer can be formed in the chlorination process. As chlorination is continued, tri-, tetra-, penta-, and hexa-chlorobenzene are formed by replacing some or all of the hydrogen atoms on the benzene ring with chlorine atoms. The pure products of $\mathrm{p}-\mathrm{DCB}$ and other polychlorobenzenes are obtained through distillation and crystallization from the mixture of polychlorinated benzenes.

Few studies on the formation of PCDD/Fs and PCBs from chlorobenzenes have been published. Buser (1979) reported significant quantities of PCDFs, a small amount of PCDDs, PCBs, and chlorophenols were formed in the pyrolysis of chlorobenzenes at $620^{\circ} \mathrm{C}$. Homogeneous gas-phase chlorination of chlorobenzene in the presence of oxygen gave substantial amounts of
PCDFs around $340^{\circ} \mathrm{C}$ (Sommeling et al., 1994). Liu et al. (2001) revealed that lower chlorinated benzenes produced more PCBs than higher ones.

Although it was noticed that PCDD/Fs maybe produced in the process of chlolorobenzenes production, no emission factor of PCDD/Fs in the production of chlorobenzenes has been given in the "Standardized Toolkit for Identification and Quantification of Dioxin and Furan Releases" (UNEP, 2003). In this paper, the occurrence and distribution of PCDD/Fs and PCBs in a process of production of $\mathrm{p}-\mathrm{DCB}$ and other chlorobenzenes were investigated. At the same time, other important intermediates were also detected in the samples. The results of this article suggest that re-estimation on the risk of using p-DCB products, especially the products for daily use such as mothballs, is expected.

\section{Experimental}

\subsection{Sampling}

A typical process of $\mathrm{p}$-DCB production in Yanzh Pesticide Co., Ltd. in Jiangsu province of China was selected in this investigation. Besides p-DCB, chlorobenzene, ortho-dichlorobenzene (o-DCB), and 1,2,4-trichlorobenzene were produced in the company.

Fig. 1 revealed that the process included several steps of chlorination and purification. Six samples, S1 to S6, were collected from the process, respectively. At first, benzene was chlorinated and a mixture (M1) of chlorobenzene and dichlorobenzenes was obtained. After chlorobenzene being distilled from $\mathrm{M} 1$, sample $\mathrm{S} 1$ was collected from the remainder mixture (M2). Then, chlorobenzene was further chlorinated to yield a mixture (M3) of dichlorobenzenes and trichlorobenzenes, and sample S2 was collected from M3. Trichlorobenzenes was separated from M3 and purified to produce the final product 1,2,4-trichlorobenzene (sample S6), while sample S3 was collected from the remainder mixture (M4). Finally, the semi-products of dichlorobenzenes (M2
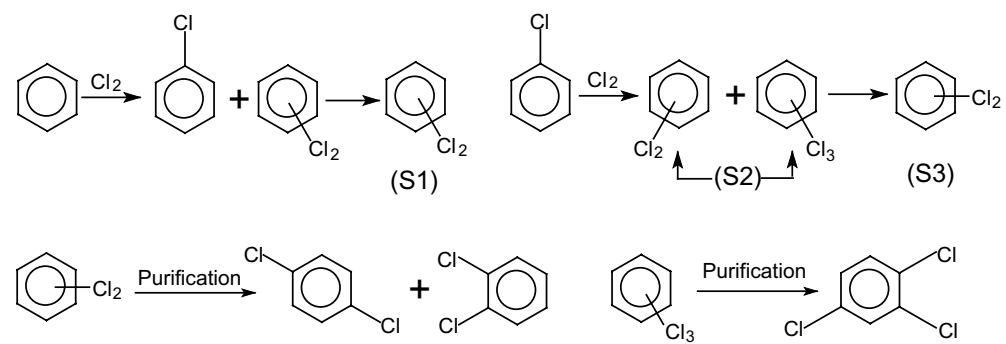

(S4)

(S5)

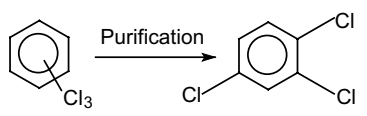

(S6)

Fig. 1. Sampling of the six samples in the process of p-DCB production. 
Table 1

Main components of the six samples

\begin{tabular}{|c|c|c|c|c|c|c|}
\hline Components & S1 $(\%)$ & $\mathrm{S} 2(\%)$ & S3 $(\%)$ & $\mathrm{S} 4(\%)$ & S5 $(\%)$ & S6 $(\%)$ \\
\hline$\overline{\mathrm{MCB}}$ & 2.0 & 3.8 & 0.62 & ND & ND & ND \\
\hline m-DCB & 0.76 & ND & ND & 1.1 & ND & 0.35 \\
\hline p-DCB & 62.7 & 66.7 & 74.3 & 98.1 & ND & 0.45 \\
\hline o-DCB & 31.7 & 26.1 & 20.7 & 0.52 & 100.0 & 0.33 \\
\hline 124-ТCВ & 2.4 & 3.0 & 3.7 & 0.31 & ND & 98.9 \\
\hline 123-TCB & 0.37 & 0.49 & 0.66 & ND & ND & ND \\
\hline
\end{tabular}

Note: MCB: chlorobenzene; DCB: dichlorobenzene; TCB: trichlorobenzene.

$\mathrm{ND}<0.1 \%$.

and M4) were merged and rectified by distillation and crystallization to produce p-DCB (sample S4) and o-DCB (sample S5). The components of the six samples are shown in Table 1 . The samples $(20 \mathrm{~g})$ were collected in April 2003 and stored at $-20^{\circ} \mathrm{C}$ until analyses were performed.

\subsection{Materials}

${ }^{13} \mathrm{C}$-labeled $\mathrm{PCDD} / \mathrm{Fs}$ and ${ }^{13} \mathrm{C}$-labeled $\mathrm{PCBs}$ were provided by Cambridge Isotope Laboratory, USA. The solvents such as $n$-hexane, toluene, and methylene dichloride were pesticide grade.

\subsection{Analysis}

After being ground and homogenized, about $0.1-1 \mathrm{~g}$ of the sample was dissolved in $n$-hexane, and ${ }^{13} \mathrm{C}$-labeled standards were added to the sample solution. Purified process was carried out by passing through an alumina column. The sample solution was concentrated finally to near dryness prior to the addition of the recovery standards. The sample was subjected to Agilent 6890 HRGC/5973N LRMS using a $30 \mathrm{~m}$
DB-5 fused-silica column for analysis. The quantification analysis of PCDD/Fs and PCBs was performed in selected ion monitoring (SIM) mode. Individual polychlorobenzene congeners in the samples were analyzed by GC/MS in SIM mode. Polychlorophenols and other intermediates were also analyzed in sample S3 to investigate the pathway of formation of $\mathrm{PCDD} / \mathrm{Fs}$ and PCBs. Polychlorophenols were derivatized with pentafluorobenzoyl chloride as a derivatizing reagent and analyzed by GC/ECD (Bao and Dong, 1990). The qualitative analysis for other intermediates was performed by GC/MS in SCAN mode. A blank ran to test for the presence of background contamination.

\section{Results and discussion}

3.1. Occurrence and distribution of PCDD/Fs in the process of p-DCB production

Table 2 presents the analytical results of 2,3,7,8$\mathrm{PCDD} / \mathrm{Fs}$ in the six samples. The average recovery was over $75 \%$.

Table 2

2,3,7,8-PCDD/Fs in the process of $\mathrm{p}-\mathrm{DCB}$ production $(\mathrm{ng} / \mathrm{g})$

\begin{tabular}{|c|c|c|c|c|c|c|}
\hline Compounds & $\mathrm{S} 1$ & $\mathrm{~S} 2$ & S3 & $\mathrm{S} 4$ & S5 & S6 \\
\hline $2378-\mathrm{T}_{4} \mathrm{CDF}$ & 1.89 & ND & ND & 0.077 & ND & ND \\
\hline $12378-\mathrm{P}_{5} \mathrm{CDF}$ & 2.43 & 10.2 & 28.5 & 0.25 & ND & ND \\
\hline $23478-\mathrm{P}_{5} \mathrm{CDF}$ & 0.35 & 0.90 & ND & ND & ND & ND \\
\hline $123478-\mathrm{H}_{6} \mathrm{CDF}$ & 1.09 & 6.96 & 14.2 & 0.14 & ND & ND \\
\hline $123678-\mathrm{H}_{6} \mathrm{CDF}$ & ND & 0.68 & 2.14 & 0.047 & ND & ND \\
\hline $234678-\mathrm{H}_{6} \mathrm{CDF}$ & 0.22 & 1.23 & 3.01 & ND & ND & ND \\
\hline $1234678-\mathrm{H}_{7} \mathrm{CDF}$ & ND & 0.22 & 0.44 & ND & ND & ND \\
\hline $1234678-\mathrm{H}_{7} \mathrm{CDD}$ & 0.17 & 0.45 & 0.063 & ND & ND & ND \\
\hline OCDD & ND & 0.14 & ND & ND & ND & ND \\
\hline OCDF & ND & ND & 0.47 & ND & ND & ND \\
\hline$\sum 2,3,7,8$-PCDDs & 0.17 & 0.59 & 0.063 & ND & ND & ND \\
\hline$\sum 2,3,7,8$-PCDFs & 5.98 & 20.0 & 48.8 & 0.52 & ND & ND \\
\hline WHO-TEQ (PCDD/Fs) & 0.62 & 1.85 & 3.37 & 0.039 & ND & ND \\
\hline
\end{tabular}

$\mathrm{ND}<0.01 \mathrm{ng} / \mathrm{g}$. 
Table 3

PCBs in the process of $\mathrm{p}-\mathrm{DCB}$ production $(\mathrm{ng} / \mathrm{g})$

\begin{tabular}{|c|c|c|c|c|c|c|}
\hline Dioxin-like PCBs & $\mathrm{S} 1$ & $\mathrm{~S} 2$ & S3 & $\mathrm{S} 4$ & S5 & S6 \\
\hline$\overline{\sum M o n o C B}$ & 2298 & 7 & 427 & ND & ND & 46.6 \\
\hline$\sum \mathrm{DiCBs}$ & 265 & 21.1 & 390 & 56.5 & 2.9 & 1.57 \\
\hline$\sum$ TriCBs & 191 & 194 & 3067 & 1717 & 19.9 & 5.72 \\
\hline$\sum$ TetraCBs & 41.4 & 163 & 663 & 23.6 & 20.6 & ND \\
\hline$\sum$ PentaCBs & 4.2 & 57.5 & 67.1 & ND & 6.0 & 161.4 \\
\hline$\sum$ HexaCBs & ND & ND & ND & ND & 1.2 & ND \\
\hline$\sum$ HeptaCBs & ND & ND & ND & ND & ND & ND \\
\hline$\sum \mathrm{PCBs}$ & 2800 & 443 & 4614 & 1797 & 50.6 & 215 \\
\hline $3,3^{\prime}, 4,4^{\prime}$-TetraCB (77) & 4.7 & 34.9 & 46.8 & 1.55 & 3.27 & ND \\
\hline $3,4,4^{\prime}, 5$-TetraCB $(81)$ & ND & ND & 4.3 & ND & ND & ND \\
\hline $2,3,3^{\prime}, 4,4^{\prime}$-PentaCB (105) & 1.9 & 9.1 & 9.3 & ND & 0.7 & ND \\
\hline $2,3^{\prime}, 4,4^{\prime} 5$-PentaCB (118) & ND & 19.8 & ND & ND & 1.24 & 14.17 \\
\hline WHO-TEQ (PCBs) & 0.0007 & 0.0064 & 0.006 & 0.0002 & 0.0005 & 0.0014 \\
\hline$\sum W H O-T E Q(P C D D / F s$ and PCBs) & 0.62 & 1.85 & 3.38 & 0.039 & 0.0005 & 0.0014 \\
\hline
\end{tabular}

$\mathrm{ND}<0.5 \mathrm{ng} / \mathrm{g}$.

$\mathrm{P}_{5} \mathrm{CDFs}$ and $\mathrm{H}_{6} \mathrm{CDFs}$ were the main congeners of PCDD/Fs as impurities, while only low levels of other $\mathrm{PCDD} / \mathrm{Fs}$ were detected in all the samples. The concentration of $\mathrm{PCDD} / \mathrm{Fs}$ in $\mathrm{S} 1$ was lower than that in $\mathrm{S} 2$ and $\mathrm{S} 3$, and the highest concentration of $\mathrm{PCDD} / \mathrm{Fs}$ was detected in S3. This hinted that the levels of $\mathrm{PCDD} / \mathrm{Fs}$ increased with the further chlorination in the process. The levels of PCDD/Fs in S4, S5 and S6 were lower than that in S1, S2 and S3. The ratios of PCDFs to PCDDs in S1, S2, and S3 were over 30, while no PCDDs were detected in S4, S5, and S6. This result is in a good agreement with previous data reported for $\mathrm{PCDD} / \mathrm{Fs}$ formation from chlorobenzenes (Buser, 1979; Sommeling et al., 1994). The contaminating levels were calculated as the TEQ values by multiplying each congener with the corresponding WHO-TEFs.

\subsection{Occurrence and distribution of PCBs in the process of p-DCB production}

The dioxin-like PCBs and the total amounts of PCBs in the samples are listed in Table 3 . The average recovery was over $75 \%$.

According to the analytical results of $\mathrm{S} 1, \mathrm{~S} 2$, and $\mathrm{S} 3$, the highest level of PCBs in S1 was monochlorobiphenyl, while that in S3 was trichlorobiphenyls. Monoand dichlorobiphenyls were the major PCBs in S1. In comparison, tri- and tetrachlorobiphenyls were the main congeners in S2 and S3. Therefore, it can be concluded that high chlorinated PCBs increased with the further chlorination.

On the basis of the data in Tables 2 and 3, although low levels of PCDD/Fs were detected in S4, S5, S6, high level of PCBs was found in them, particularly in S4.
Apparently, PCDD/Fs decreased obviously in the purification process, but PCBs cannot be removed effectively. It also can be seen that levels of PCBs in S4, S5, and S6 were different, which maybe due to the differences of the purification procedures.

The production process of $\mathrm{p}-\mathrm{DCB}$ was similar to that of PCBs. PCBs were manufactured commercially by the progressive chlorination of biphenyl in the presence of a suitable catalyst, e.g., iron chloride. In general, commercial PCBs contained PCDFs in the $\mu \mathrm{g} /$ $\mathrm{kg}$ to $\mathrm{mg} / \mathrm{kg}$ range but no PCDDs (UNEP, 2003). Considering above, the detection of PCDFs and PCBs in the process of $\mathrm{p}-\mathrm{DCB}$ production is reasonable.

In addition, since semi-manufactured products contained relative high levels of $\mathrm{PCDD} / \mathrm{Fs}$ and PCBs, it is very important to clean up impurities in the process. Moreover, it is obvious that high level of $\mathrm{PCDD} / \mathrm{Fs}$ and PCBs still remain in the residue of the production. Thus, the residue should be treated according to the guideline of disposal of hazard waste.

\subsection{The significance of finding of Polychlorophenols, Polychlorinated biphenylethers (PCBEs) and Polychlo- rinated phenylphenols (PCPPs) in the process of p-DCB production}

Table 4 presents the analytical results of polychlorophenols in sample S3. Ortho-chlorophenol, 2,4-dichlorophenol, 2,4,6-trichlorophenol and 2,3,4,6- tetrachlorophenol were the main congeners of polychlorophenols. The common character of these polychlorophenols was ortho- and para-position of the hydroxyl substituted by chlorine, for the ortho- and para-position substituted 
congeners are easier to be formed from polychlorobenzenes in the presence of oxygen. Weber and Hagenmaier (1999) has investigated the importance of chlorophenols in the formation of PCDD/Fs. They concluded that polychlorinated dihydroxybiphenyls and ortho-phenoxyphenols were the key intermediates in the formation of PCDFs from chlorophenols. It should be emphasized, however, that the reaction pathway involving condensation of chlorophenols via dioxaspiro intermediates mainly leaded to the formation of PCDDs (Tuppurainen et al., 1998, 2000), while PCDFs are probably involved other precursors such as polychlorinated biphenylethers (PCBEs) and polychlorinated phenylphenols (PCPPs).

Qualitative analysis of some compounds was performed to further study the pathway of PCDD/Fs formation. As a result, two important possible precursors, 1,4-dichloro-2-phenoxybenzene (Fig. 2) and 2-phenyl2',5,5'-trichlorophenol (Fig. 3), were found in sample S3. These compounds could possibly serve as reaction intermediates in the formation of PCDD/Fs from chlorobenzenes. In addition to the two intermediates, other chlorinated compounds such as tetrachlorobenzenes, 3,4,5,6-tetrachlorocyclohexene, pentachlorocyclohexene, biphenyl, and terphenyl were also detected in sample S3.

On the basis of this study and the previous research works (Babushok and Tsang, 2003; Evans and Dellinger, 2003), a pathway of the formation of PCDD/Fs and PCBs from polychlorobenzenes in the presence of chlorine is shown in Fig. 4. Previous studies have revealed that PCBEs (species 5, e.g. 1,4dichloro-2-phenoxybenzene), PCPPs (species 2, e.g. 2phenyl-2',5,5'-trichlorophenol), PCBs (species 1) and polychlorophenols (species 4) were the important precursors in the formation of $\mathrm{PCDD} / \mathrm{Fs}$ (Sommeling et al., 1994; Tuppurainen et al., 1998). As seen in Fig. 4, chlorine atoms abstract $\mathrm{H}$ from chlorobenzenes, the resulting polychlorophenyl radicals react rapidly with chlorobenzenes, $\mathrm{Cl}_{2}$, or $\mathrm{O}_{2}$ (Sommeling et al., 1994). The reaction with chlorobenzenes leads to PCBs. PCBs can be oxidized and PCDFs are formed via PCPP radicals or PCPPs. The reaction with $\mathrm{Cl}_{2}$ produces polychlorobenzenes. The third reaction with $\mathrm{O}_{2}$ leads to chlorophenoxyl radicals and $\mathrm{PCDD} / \mathrm{Fs}$ are formed finally (Ballschmiter et al., 1983; Sommeling et al., 1994). A reaction of polychlorophenols or polychlorophenol radicals with polychlorobenzenes could lead to PCBEs, which are known to form PCDFs (and to a lesser degree PCDDs) upon pyrolysis (Buser, 1979). In the pathway mentioned above, PCDFs can be formed via some intermediates, e.g. PCBEs, and PCPP radicals, while PCDDs are formed only via PCBEs. This perhaps results in more PCDFs and less PCDDs detected in the samples. 


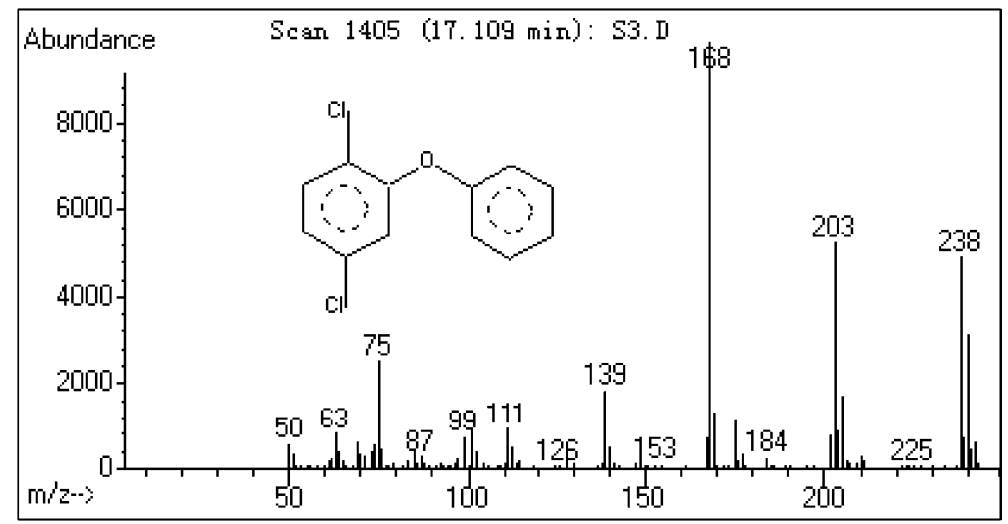

Fig. 2. Mass spectrum of 1,4-dichloro-2-phenoxybenzene.

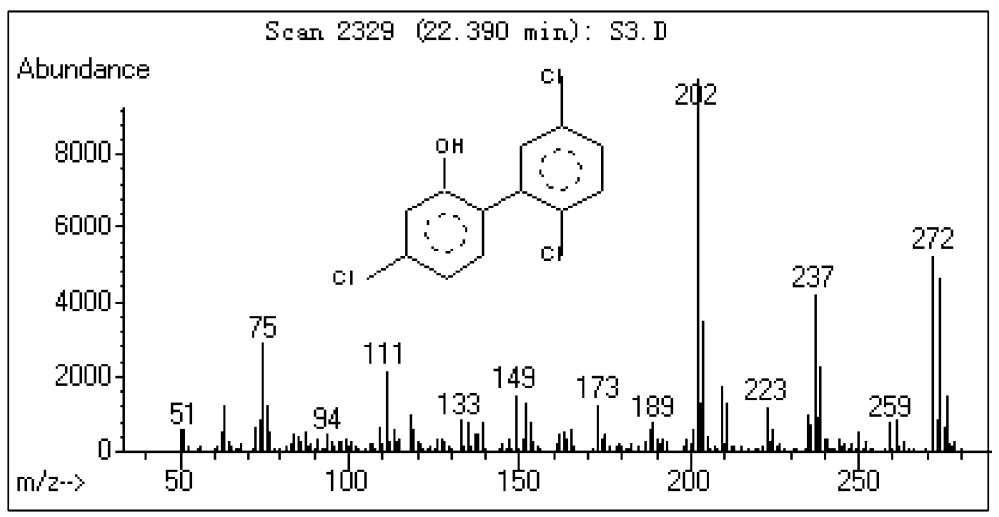

Fig. 3. Mass spectrum of 2-phenyl-2',5,5'-trichlorophenol.

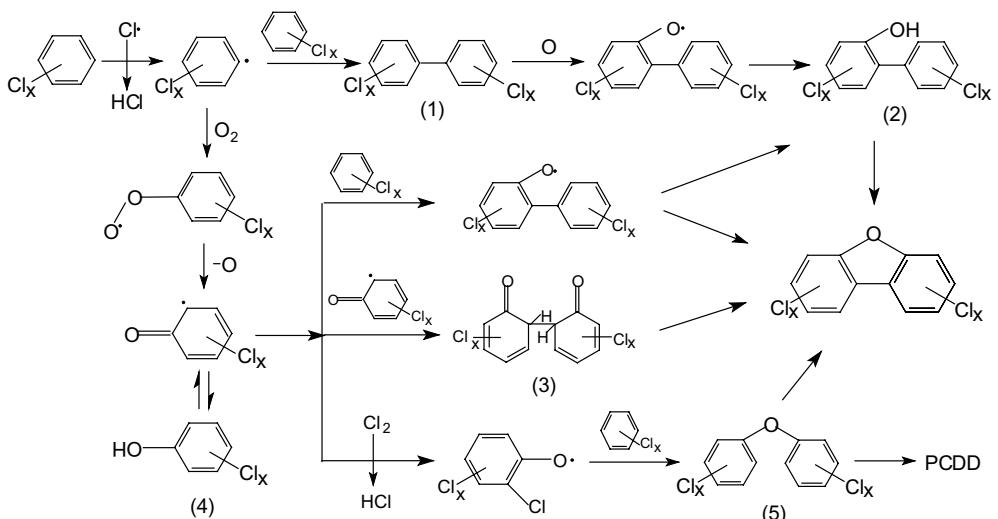

Fig. 4. Schematic picture representation of PCDD/Fs and PCBs formation routes $(x=0-6)$.

\section{Acknowledgments}

This study is supported by National 973 Project (2003CB415006) and National 863 Project (2002AA641010).

\section{References}

Babushok, V.I., Tsang, W., 2003. Gas-phase mechanism for dioxin formation. Chemosphere 51, 10231029. 
Ballschmiter, K., Zoller, W., Scholz, C., Nottrodt, A., 1983. Occurrence and absence of polychlorodibenzofurans and polychlorodibenzodioxins in fly ash from municipal incinerators. Chemosphere 12, 585-594.

Bao, Z., Dong, J., 1990. Gas chromatographic determination of phenols in water by capillary GC/ECD. Chin. Environ. Chem. 9 (2), 26-33.

Buser, H.R., 1979. Formation of polychlorinated dibenzofurans and dibenzo-p-dioxins from the pyrolysis of chlorobenzenes. Chemosphere 6, 415-424.

Evans, C.S., Dellinger, B., 2003. Mechanisms of dioxin formation from the high-temperature pyrolysis of 2-chlorophenol. Environ. Sci. Technol. 37 (7), 1325-1330.

HSDB, 1998. Hazardous Substances Data Bank (1,4 dichlorobenzene). National library of medicine. National toxicology program. Bethesda, MD.

Liu, P.-Y., Zheng, M.-H., Zhang, B., Xu, X.-B., 2001. Mechanism of PCBs formation from the pyrolysis of chlorobenzenes. Chemosphere 43, 783-785.

Sommeling, P.M., Mulder, P., Louw, R., 1994. Formation of PCDFs during chlorination and oxidation of chlorobenzene in chlorine/oxygen mixtures around $340^{\circ} \mathrm{C}$. Chemosphere 29, 2015-2018.

Tuppurainen, K., Halonen, I., Ruokojärvi, P., Tarhanen, J., Ruuskanen, J., 1998. Formation of PCDDs and PCDFs in municipal waste incineration and its inhibition mechanisms; a review. Chemosphere 36, 1493-1511.

Tuppurainen, K.A., Ruokojärvi, P.H., Asikainen, A.H., Aatamila, M., Ruuskanen, J., 2000. Chlorophenols as precursors of PCDD/Fs in incineration processes: correlations, PLS modeling, and reaction mechanisms. Environ. Sci. Technol. 34 (23), 4958-4962.

UNEP, 2003. UNITED NATIONS, ENVIRONMENT PROGRAMME, Standardized Toolkit for Identification and Quantification of Dioxin and Furan Releases, first ed., Prepared by UNEP, Chemicals Geneva, Switzerland.

Weber, R., Hagenmaier, H., 1999. Mechanism of the formation of polychlorinated dibenzo-p-dioxins and dibenzofurans from chlorophenols in gas phase reactions. Chemosphere $38,529-549$. 